\title{
Microwave Measurements of the Complex Permittivity of Zirconia at High Temperatures
}

\author{
Thoria A. Baeraky \\ Faculty of Science, Physics Department, \\ King Abdulaziz University, Jeddah, Saudi Arabia
}

The real and the imaginary parts of the complex permittivity of zirconia were measured at frequencies from 614.39 to $3820.66 \mathrm{MHz}$ and over the temperature range $20-1800{ }^{\circ} \mathrm{C}$. Two systems of the cavity perturbation techniques were used, one in King Abdulaziz, University Saudi Arabia, and the other in the University of Nottingham, United Kingdom. The electrical conductivity and the activation energy of zirconia were calculated from these measurements. The conduction mechanisms of zirconia, at high temperatures in the microwave region, have been studied using these calculations.

\section{Introduction:}

Microwave processing is a field of increasing importance and is receiving more attention particularly in processing materials with a broad range of compositions, sizes and shapes. [1-3]. In recent years microwave processing research and development have been expanded into many new areas such as ceramics, polymers, composites, and chemicals [4-6]. Thermal runaway, the sudden rise of temperature during the microwave heating, and a uniform heating are the challenges facing the controlling of microwave processing of materials. It was shown that the complex permittivity of material must be known to control the microwave processing of ceramics [7]. The real and imaginary parts of the complex permittivity, $\varepsilon$ ' and $\varepsilon$ ", are parameters describe the behaviour of a dielectric material under the influence of a microwave field. Both affect the power absorbed and the half-power depth. They describe how microwaves penetrate and propagate through an absorbing material, and they influence the volumetric heating of a given material [8]. Ceramics are inorganic materials of wide usage particularly when used as the elements of microwave microcircuits [9]. Their great advantage is that they enable the reduction of size of the components and the relatively low cost in the production of a variety of microwave devices such as oscillators and filters [10]. Fine ceramics, which are excellent in resistance to heat and corrosion, are promising as structural 
materials capable of replacing metal under high-temperature use [11]. Cavity perturbation technique is the best method of measuring the dielectric properties of ceramics in the microwave range of frqeuncies. It is distinguished by its higher measuring precision and simple calculations and does not have a special requirement for one geometry, size and kind of the sample such as solid, powder, and liquid [12].

Zirconia, the compounds of metals with oxygen ions $\left(\mathrm{ZrO}_{2}\right)$, is an oxide ceramic [13]. Pure crystalline zirconia is a transformation material with different crystal structures of the same chemical composition. It is in a monoclinic $\left(\mathrm{T}<1200{ }^{\circ} \mathrm{C}\right)$, tetragonal $\left(1200{ }^{\circ} \mathrm{C}<\mathrm{T}<2200{ }^{\circ} \mathrm{C}\right)$, or cubic structure $\left(\mathrm{T}>2200{ }^{\circ} \mathrm{C}\right)$ [14]. The transition in zirconia is displacive transformation, which involves distortion of the structure, such as a change in bond angles, but does not include breaking of bonds. During heat-up this transformation is known as transformation toughening which refers to increase in fracture toughness achieved by dispersion of particles of a material that undergoes a displacive transformation in a matrix material that does not go through the same transformation. During the cooling cycle, after initial densification of the material, the dispersed particles undergo transformation that is accompanied by a volume change. $\mathrm{ZrO}_{2}$ undergoes a $3.25 \%$ volume expansion during cooling below approximately $1000{ }^{\circ} \mathrm{C}$ due to transformation from the tetragonal phase to the monoclinic phase which results in very high internal stresses at the grain boundaries and many cracks are initiated which lead to material destruction [15]. This undesirable phase transformation can be eliminated by stabilization of the cubic phase with an addition of $\mathrm{CaO}, \mathrm{MgO}$, or $\mathrm{Y}_{2} \mathrm{O}_{3}$ and when controlled this addition will produce a stable cubic form of $\mathrm{ZrO}_{2}$ that does not undergo a transformation [16].

In this work the real and imaginary parts, $\varepsilon$ ' and $\varepsilon$ "' of the complex permittivity, of pure zirconia, $\mathrm{ZrO}_{2}$, was measured at high temperatures in the microwave region using the cavity perturbation technique.

\section{Experimental:}

Cavity perturbation is the technique which was used for these measurements. The details of this technique have been reported in [17]. The measurements of $\varepsilon$ ' and $\varepsilon$ ', of the same material, pure zirconia, $\mathrm{ZrO}_{2}$, were repeated using the same system in Nottingham University. The effect of the silica tubes, the holders of the sample powder, was considered and removed. Five tubes of $2,3,4,5$, and $6 \mathrm{~mm}$ in diameter were used to hold the samples, which were moved between the furnace, and the cavity. The effect of these tubes on the shift of frequency and Q-factor, was removed by heating those tubes, without the samples, from $25{ }^{\circ} \mathrm{C}$ up to $1800{ }^{\circ} \mathrm{C}$ in steps of $50{ }^{\circ} \mathrm{C}$ and 
subtracting those shifts from the shift of frequencies and Q-factor which was produced by the tube filled by the sample in the same step of temperature. The general perturbation equations where the real, $\varepsilon$, and imaginary, $\varepsilon^{\prime \prime}$, parts of the complex permittivity were measured in terms of the real and imaginary parts of the frequency shift have been derived as [18]:

where

$$
\begin{aligned}
& \varepsilon^{\prime}-1=2 J_{1}^{2}\left(x_{0 n}\right) \frac{\Delta f}{f_{0}} R_{o}^{2} / R_{s}^{2} \\
& \varepsilon^{\prime \prime}=j_{1}^{2}\left(\chi_{0 n}\right) \Delta\left(1 / Q_{L}\right) R_{o}^{2} / R_{s}^{2}
\end{aligned}
$$

and

$$
\Delta f=f_{o}-f_{1}
$$

$$
\Delta\left(1 / Q_{L}\right)=\left(\frac{1}{Q_{L 1}}-\frac{1}{Q_{L o}}\right)
$$

$\mathrm{f}_{\mathrm{o}} \quad$ resonant frequency;

$\mathrm{f}_{\mathrm{s}} \quad$ resonant frequency with test sample;

$Q_{\text {Lo }} \quad$ loaded cavity Q-factor of empty cavity;

$Q_{\mathrm{L} 1} \quad$ loaded cavity Q-factor of cavity with test sample;

$R_{o} \quad$ radius of cavity;

$R_{S} \quad$ radius of sample;

$\mathrm{x}_{\text {on }} \quad \mathrm{n}^{\text {th }}$ root of $J_{\mathrm{o}}(\mathrm{x})=0$;

$\varepsilon^{\prime}$ and $\varepsilon^{\prime \prime} \quad$ respectively, real and imaginary parts of the complex permittivity calculated by the simple perturbation theory.

\section{Results and Discussion:}

The two components of the complex permittivity, i.e. real and imaginary parts, $\varepsilon^{\prime}$ and $\varepsilon^{\prime \prime}$, of pure zirconia, $\mathrm{ZrO}_{2}$ were measured from 25 to $1800{ }^{\circ} \mathrm{C}$ and from 614.39 to $3820.66 \mathrm{MHz}$ in temperature and frequency ranges respectively. The temperature and frequency-dependent conductivity have been investigated in terms of the activation energy, $E_{A}$, and the frequency exponent $n$, of the universal power law. The variation of these parameters will be interpreted as a function of temperature and frequency in order to determine whether the conduction mechanisms of these materials at high temperature in the microwave range are fundamentally different from those determined from low frequency measurements done at room temperature. 

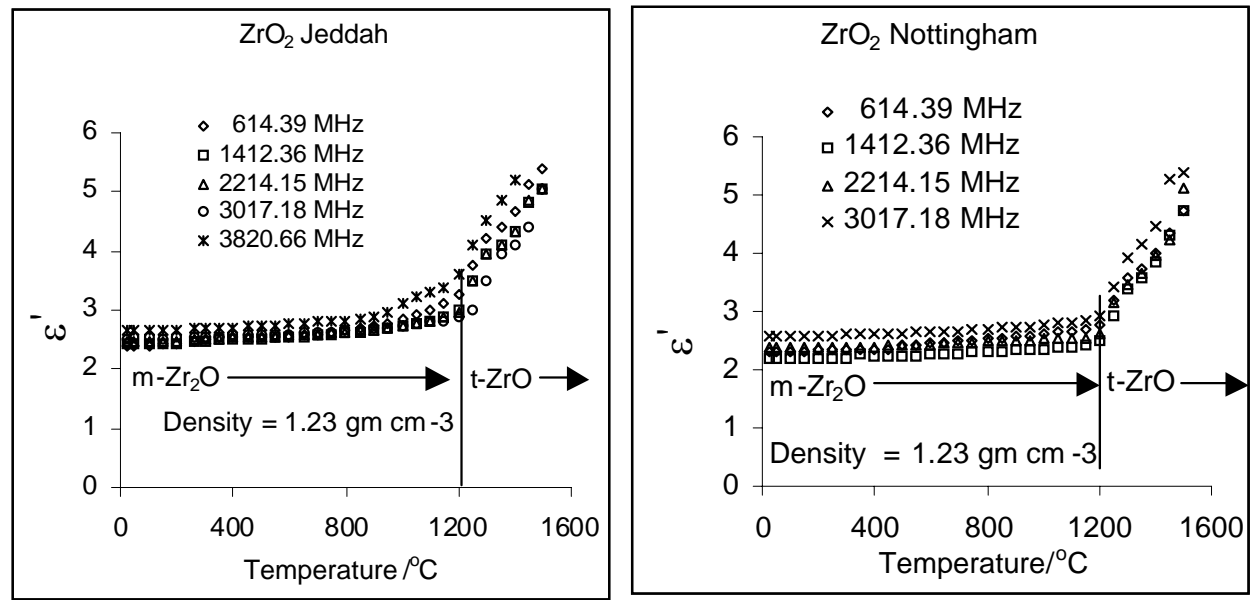

Fig. (1): Pure zirconia complex permittivity real parte' vs. temperate and frequency.
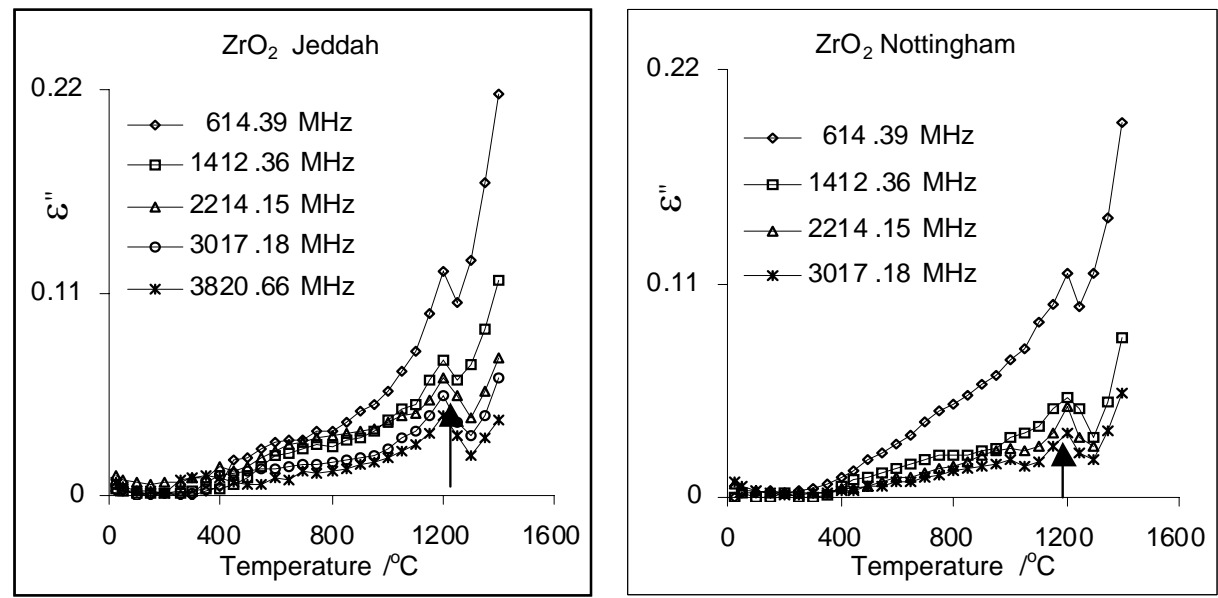

Fig. (2): Pure zirconia complex permittivity imaginary part $\varepsilon^{\prime \prime} \mathrm{Fm}^{-1}$ vs. temperature and frequency.

The abrupt large increase in the real part of the permittivity, $\varepsilon^{\prime}$, at the temperature $1200{ }^{\circ} \mathrm{C}$, shown in Fig. (1) emphasises that this change is due to the phase transformation of zirconia from the monoclinic phase to tetragonal phase where the variation of $\varepsilon^{\prime}$ below $1200{ }^{\circ} \mathrm{C}$, is a negligible variation with temperature. A larger increase in $\varepsilon^{\prime}$ at the point of transition is due to the sudden decrease in the unit cell volume of about $5 \%$ with monoclinic to tetragonal transformation [19]. The other guide to the phase transition of zirconia $1200{ }^{\circ} \mathrm{C}$ is the sudden decrease in the imaginary part of the permittivity, $\varepsilon$ ", shown in Fig. 2. These figures show that the real and the imaginary parts measurements are identical for the two measurement systems of Jeddah and Nottingham and 
there is a good agreement with the increasing of the complex permittivity two parts, $\varepsilon^{\prime}$ and $\varepsilon^{\prime \prime}$, at the phase transformation with the measurements of pure zirconia. Which, was measured by Hutcheon [20].

The conduction mechanism of pure zirconia was investigated in terms of the measured permittivity imaginary part, $\varepsilon$ ", and the corresponding frequency, $\omega$, of eqn. (3) was used to calculate the electrical conductivity, $\sigma$ in order to determine the frequency exponent, $n$. The activation energy, $E_{\mathrm{A}}$, was found by using the slopes of equation (4) and (5) respectively [21].

$$
\begin{aligned}
& \sigma(\omega)=\varepsilon_{o} \omega \varepsilon^{\prime \prime}(\omega) \\
& \sigma(\omega)=A \omega^{n} \\
& \sigma=A \exp \left(-E_{A} / K T\right)
\end{aligned}
$$

Where $\sigma$ is the electrical conductivity, $E_{a}$ is the activation energy in $\mathrm{eV}, k$ is Boltzmann constant $\left(k=8.617 \times 10^{-5} \mathrm{eV} / \mathrm{K}\right)$, and $\mathrm{T}$ is the absolute temperature/ K. The relation of $\log \sigma(\omega)$ vs. $10^{3} /$ Tis illustrated in Fig. 3 for the two system measurements of Jeddah and Nottingham which will be discussed later.
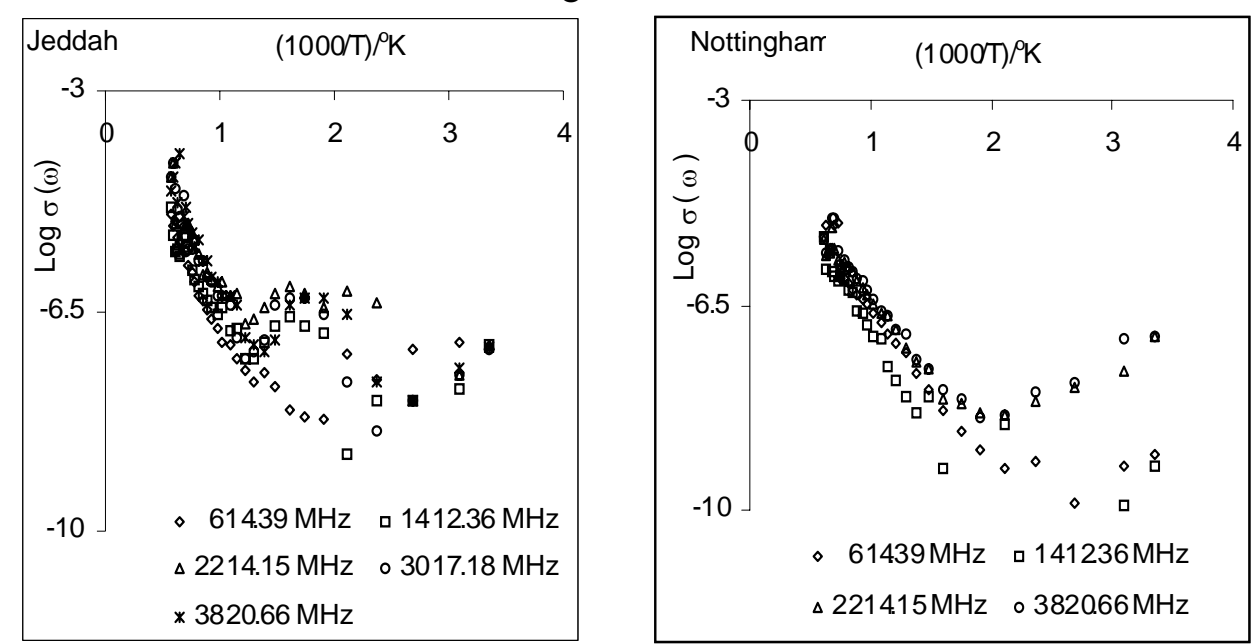

Fig. (3): Electrical conductivity of zirconia vs. frequency and the reciprocal temperature.

From conductivity measurements it was able to calculate the activation energy $\left(E_{\mathrm{A}}\right)$ variation with temperature at different frequencies using Eqns. (3) - (5). The results are shown in Fig. 4; indicating reasonable agreement with electrical conductivity variations in Fig. 5. 


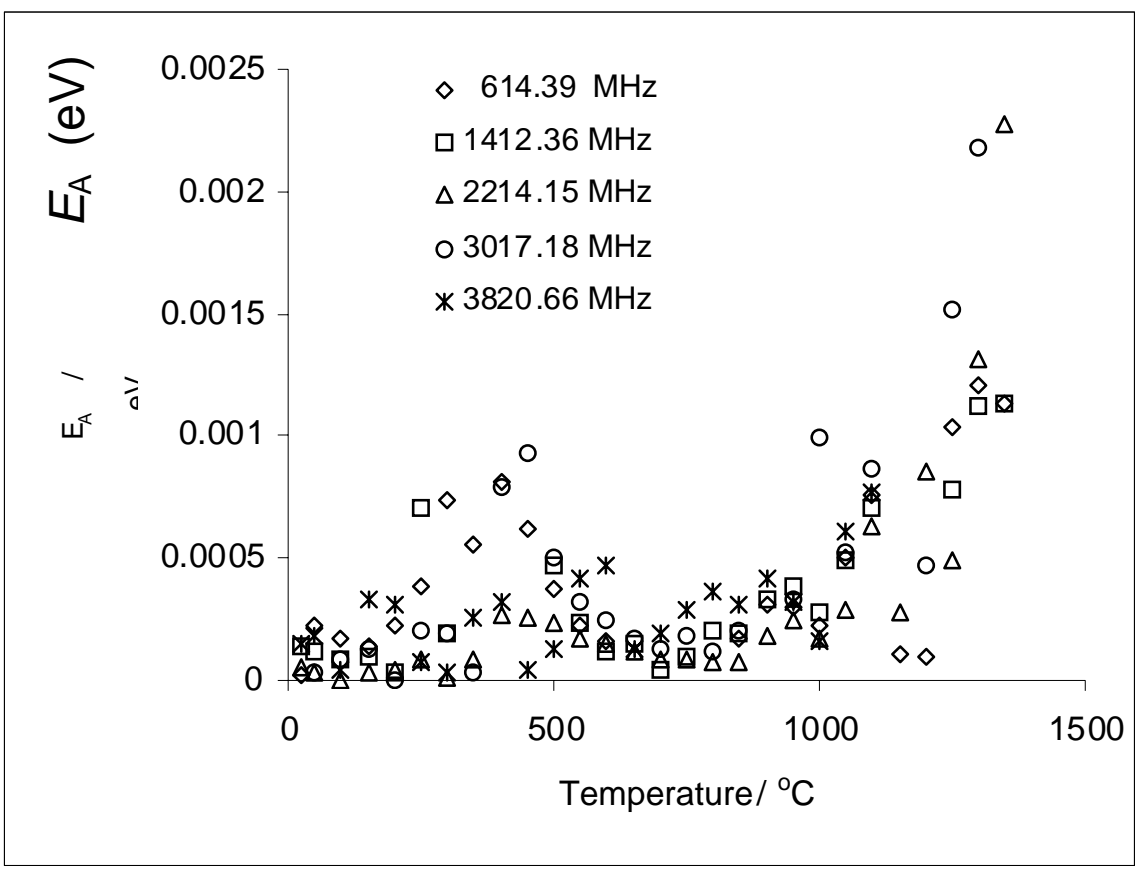

Fig. (4): Activation energy variation with temperature at different microwave frequencies.

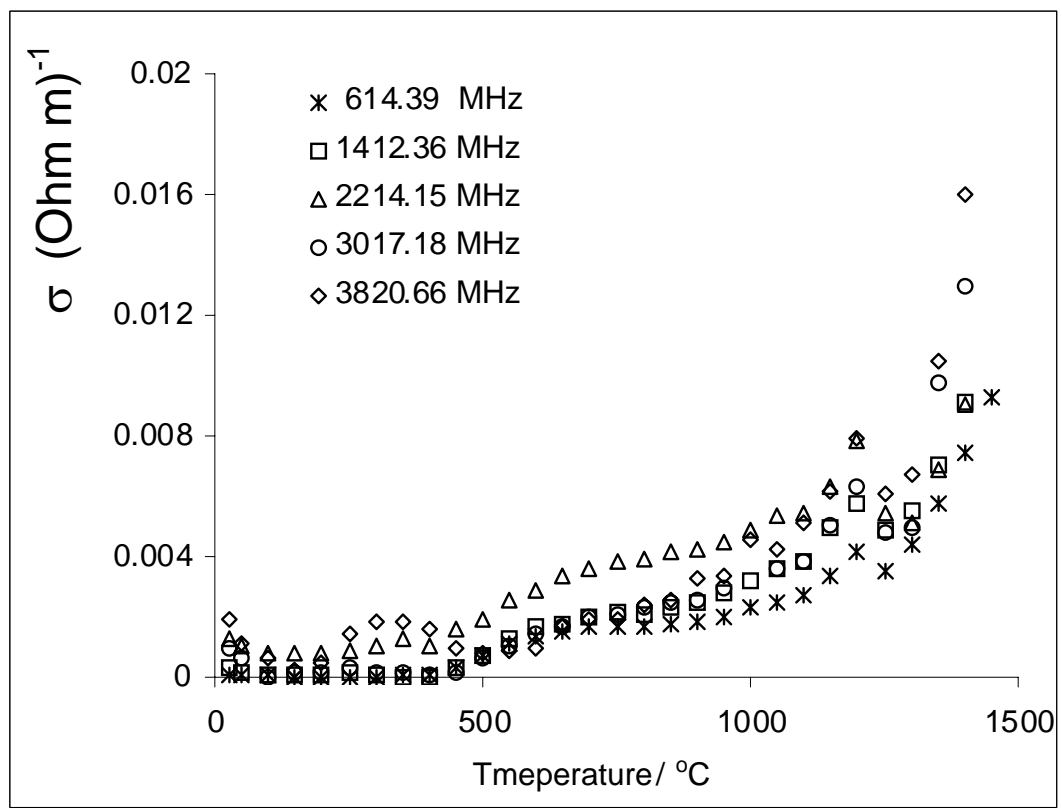

Fig. (5): Electrical conductivity variation with temperature at different microwave frequencies. 
The frequency dependence of the electrical conductivity of pure zirconia was investigated in terms of the frequency exponent $n$ of Eqn. (4). This exponent was calculated by using the slope of $\log \sigma$ vs. $\log \omega$ shown in Fig. 3 and its relation with temperature is illustrated in Fig. 6.

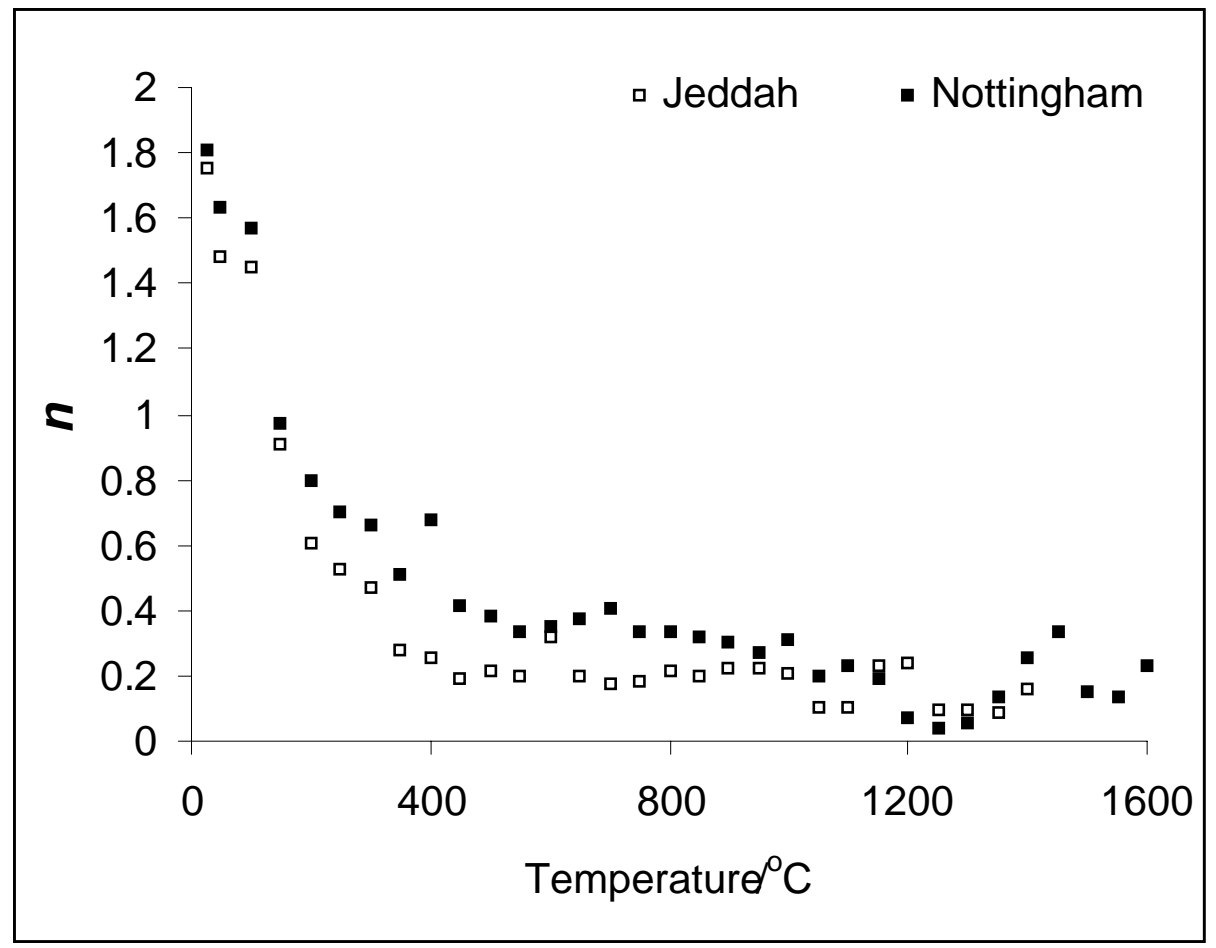

Fig. (6): The temperature dependence of the frequency exponent, n, of pure zirconia

The data presented in Figs. 1-6. show that for pure zirconia there are three regions of the electrical conductivity variation with temperature at difference frequencies. These regions can be classified as: $\mathrm{T}<400{ }^{\circ} \mathrm{C}, 1200{ }^{\circ} \mathrm{C}$ $>\mathrm{T}>400{ }^{\circ} \mathrm{C}$, and $\mathrm{T}>1200{ }^{\circ} \mathrm{C}$. As discussed above the change in behavior at $1200{ }^{\circ} \mathrm{C}$ can be related to the tetragonal phase transition.

In the temperature region, $\mathrm{T}<400{ }^{\circ} \mathrm{C}$, the conductivity and the activation energy shown in Figs. 3 - 5, are strongly frequency but weakly temperature dependent. The universal conductivity curve of Fig. 6 in the temperature region $\mathrm{T}<400{ }^{\circ} \mathrm{C}$ has a shape which is in agreement with that observed by Greenacre for Yttria doped Zirconia [22] with $n$ increasing through 1 as the temperature falls. This leads to the consideration that the conduction in pure zirconia below $400{ }^{\circ} \mathrm{C}$ is ionic conduction. 
The temperature region $400{ }^{\circ} \mathrm{C}<\mathrm{T}<1200{ }^{\circ} \mathrm{C}$ in Fig. 3 shows that the $\log \sigma$ is almost a linear function of $10^{3} / \mathrm{T}$ and its deviations from linearity at the temperature region, $\mathrm{T}<400{ }^{\circ} \mathrm{C}$, have been neglected. The permittivity, the conductivity, and the activation energy of $\mathrm{ZrO}_{2}$, shown in Figs. 1-5, are very weakly temperature dependent and strongly frequency dependent. The exponent $n$ shown in Fig. 6 is nearly constant in the temperature region, $400{ }^{\circ} \mathrm{C}<\mathrm{T}<$ $1200{ }^{\circ} \mathrm{C}$, which emphasizes the temperature and frequency dependence of pure zirconia in temperature region $400{ }^{\circ} \mathrm{C}<\mathrm{T}<1200{ }^{\circ} \mathrm{C}$ discussed in the previous paragraph. Kawamura et al [23] have shown that in pure zirconia the interatomic bonds are such that ionic and electronic semiconductors coexist; in the case of a highly ionic bond electrons remain attached to the nuclei until high temperatures are reached then the electronic conductivity is generally superior to the ion conductivity. This leads to the possibility that conduction in monoclinic pure zirconia of microwave frequencies before the phase transformation at high temperature, $\mathrm{T}<1200{ }^{\circ} \mathrm{C}$, is due to the contribution of both ions and electrons.

The conductivity and the activation energy dependence on temperature and frequency in the third temperature region, $\mathrm{T}>1200{ }^{\circ} \mathrm{C}$ after the transition, becomes more complicated to observe, as shown in Figs. $3-5$. The conduction mechanism in this region is unclear and it may be considered as the metallic conduction according to the Mott transition [24]. It is a semiconductor-metallic phase transition, which takes place when a conduction and a valence bands are caused to overlap as a result of the change in the composition or the specific volume of the material with increasing temperature. This was observed in the monoclinic to tetragonal phase transition in vanadium dioxide, $\mathrm{VO}_{2}$, at $340 \mathrm{~K}$ [25]. Zirconia undergoes a similar transition at $1200{ }^{\circ} \mathrm{C}$ but there is an extreme increas of its activation energy after transition, as shown in Fig. 5. This indicated that the overlap between the conduction and valence bands is not complete with zirconia as in the case of $\mathrm{VO}_{2}$.

\section{Conclusion:}

The well-known phase transformation from monoclinic to tetragonal was observed in pure zirconia during the measurement of the complex permittivity. The conduction mechanism and the related loss of pure zirconia has been observed in terms of the imaginary part, $\varepsilon^{\prime \prime}$, the activation energy, and the electrical conductivity. Studying the conduction mechanism of pure zirconia in terms of the complex permittivity measurements leads to conclusion that the conduction mechanism of pure zirconia changed from ionic to electronic in the microwave frequency range at high temperatures. The above conclusion emphasised that the complex permittivity is important in understanding the interaction between the electromagnetic waves and ceramic 
material. This is particularly important for the microwave processing of ceramics controlled.

\section{References:}

1. W. H. Sutton, J. Mat. Res. Soc, 269, 3, (1992).

2. J. G. P. Binner, T. E. Cross, J. Mat. Res. Soc,. 269, 33., (1992).

3. R. D. Agrawal, J. P. Cheng, M. Mathis, Ceramic Transactions (Microwaves).., 80, 3, (1997).

4. I. Ahmad, D. E. Clark, Ceramic Transactions Microwaves, 21, 605, (1991).

5. R. Gedye, K. Westaway, F. Smith, Ceramic Transactions Microwaves, 59, 525, (1995).

6. J. Buckley, R. Bryant, M. Long, A. Buchman, J. R. Gleason J. Ceram. Soc., 80, 499, (1997).

7. X. D. Yu, V. V. Varadan, V. K. Varadan, D. Ghodgaonkar, J. Ceram. Soc., 21, 251, (1991).

8. W. H. Sutton, J. Am. Ceram. Soc. Bull. , 68(2), 376, (1989).

9. J. H. Morris, Y. Belopolsky, J. Proceeding of the SPIE-The International Society for Optical Engineering. 1389, 236, (1990).

10. J. M. Wu, M. C. Chang, P. C. Yao, J. Ceram. Soc., 73(6), 1599, (1990).

11. Y. Ishikawa, H. Tamura, T. Nishikawa, K. Wakino, J. Ferroelectrics, 135, 371, (1992).

12. Z. Jian, Ch. Jiping, T. Yuling, Q. Jinyu, J. Mat. Res. Soc,. 430, 263, (1996).

13. J. B. Wachtman, "Mechanical Properties of Ceramics", John Wiley and Sons, INC. (1996).

14. F. A. Kroger, Ceramic Transactions Microwaves Processing, 49 (4), 216, (1966).

15. D. W. Richerson, "Modern Ceramic Engineering", Marcel Dekker Inc. New York, 1982.

16. D. Kim, H. Jung, J. Jang, H. Lee, J. Ceram. Soc., 81(9), 2309, (1998).

17. T. A. Baeraky, Egypt. J. Sol., 25 (2), 263, (2002).

18. S. Li, C. Akyel, R. G. Bosisio, J. IEEE MTT, 29 (10), 230, (1981).

19. D. P. Thompson, A. M. Dikins, J. Mat. sci., Vol. 27, p. 2267, 1992.

20. R. M. Hutcheno, P. Hayward, B. H. Smith, S. B. Alexander, J. Ceram. Soc., 59, 235, (1995).

21. D. W. Strickler, W. G. Carlson, J. Ceram. Soc., 47, 122, (1984).

22. N. R. Greenacre, PhD thesis, University of Nottingham, UK, (1997).

23. K. Kawamura, K. Watanabe, Y. Nigara, A. Kaimai, T. Kawaba, J. Mizusaki, J. Electrochem. Soc., 145(7), 2552, (1998). 
24. N. F. Mott, "Conduction in Non - crystalline materials" Taylor \& Francis, New York, (1990). 